\title{
Designing effective educational games - a case study of a project management game
}

\author{
Jakub Miler, Agnieszka Landowska \\ Gdansk University of Technology, \\ Faculty of Electronics, Telecommunications and Informatics, \\ Narutowicza Street 11/12, 80-233, Gdansk, Poland \\ Email: \{jakubm, nailie\}@eti.pg.gda.pl
}

\begin{abstract}
This paper addresses the issues of designing effective educational games. We aim at investigating how the cognitive, behavioral and emotional aspects of the games influence their educational effectiveness. The results were obtained with an observational user experience study extended with affect analysis carried out for a project management game GraPM. We analyzed the players' understanding of the game mechanics and logic, their engagement and emotional state. Then we confronted it with the educational effects achieved. In this case study the key identified factors of educational effectiveness were: understanding game mechanics, player's engagement, and feeling of control. Invoking other desired emotions was not required for effective education, which was also generally unrelated to the player's initial knowledge.
\end{abstract}

\section{INTRODUCTION}

$\mathrm{E}$ DUCATION has always been an important part of human's activity. Effective education requires satisfaction of multiple goals according to Bloom's taxonomy and its newer revisions [1]. One of the recognized tools to provide multi-layer educational experience are games, with computer games in particular. The use of games in education is additionally promoted by the "serious games" approach [2].

Playability is a commonly used term for computer games, however it can be evaluated the same way as usability [3]. Usability of software as defined by ISO 9241 standard includes effectiveness, efficiency, and satisfaction with which specified users achieve specified goals in particular environments [4]. User experience (UX) extends traditional usability with affective aspects forming more holistic picture of human-system interaction.

The evaluation of user experience is particularly important for educational games as the cognitive, behavioral and emotional aspects of a game can largely influence its educational effectiveness. Our research goal is to understand the factors that influence the educational effectiveness of computer games and utilize it to design better educational

This work was supported in part by Polish-Norwegian Financial Mechanism Small Grant Scheme under the contract no. PolNor/209260/108/2015 as well as by DS Funds of ETI Faculty, Gdansk University of Technology. games. This leads to the following research questions of this paper: (RQ1) how usability of a game affects its educational effects? (RQ2) how user experience of a game affects its educational effects?

This paper presents an extended user experience study of an educational game GraPM and is organized as follows. Section II discusses the related work. Section III describes briefly the game under study and its usability goals. Sections IV and $\mathrm{V}$ provide the study plan and results, respectively, followed by the concluding remarks in section VI.

\section{RELATED WORK}

The related work for our study can be divided into two main topics: (1) educational games; (2) usability and user experience research.

We are mostly interested in the management games in general and project management games in particular. The example project management training games are Symulator Projektu and Symulator Zachowań Menadżerskich from Grupa ODiTK [5], That Project Management Game [6], or Scrum Game [7]. No usability, user experience or educational effectiveness studies for most of these games were identified. Scrum Game has been evaluated preliminarily, but only with a simple questionnaire.

Broad discussions of the issues of game usability are presented in [8], but they do not address specifically the educational games. P. Mirza-babaei introduced biometrics to evaluate the gameplay experience, but applied it to a noneducational FPS-type game [9]. Parodi et al. measured the player's education with their Competence Performance Analyser tool with limited use of UX and biometrics [10]. Raabe, Santos, Paludo, and Benitti carried out an extensive evaluation of serious games teaching project management, however the evaluation did not include the usability [11].

Research on both user experience and affective computing is broad and have already been summarized several times, e.g. in the work of Vermeen et al. [12] or the book by Albert and Tullis [13]. There are a few studies on fusing affect recognition and usability evaluation [14]-[16]. The most important work is the one by Ahn and Picard, that proposed the Affective-Behavioral-Cognitive $(\mathrm{ABC})$ framework for 
the user experience evaluation. The framework was validated with an experiment on beverages [14]. Lew, Olsina, Becker, and Zhang applied the affect evaluation in the quality assurance procedures for web applications [15]. Kołakowska, Landowska, Szwoch, Szwoch, and Wróbel proposed the application of affect recognition in usability evaluation in four different scenarios: first impression test, task-based usability test, free interaction test and comparative test [16].

\section{THE GAME UNDER STUDY}

We carried out this research with a project management educational game GraPM, which was conceived and designed by J. Miler based on his earlier research on project management [17]-[18]. GraPM stands for "game on project management", where "gra" is game in Polish. This game puts the player in the role of a project manager. The target group of this game includes: (1) people who want to increase their project management knowledge and skills; (2) players who like management and strategy games.

The GraPM game was implemented within an engineering diploma project in 2014 [19]. It is a JavaScript-based rich internet application run in a web browser, see Fig. 1.

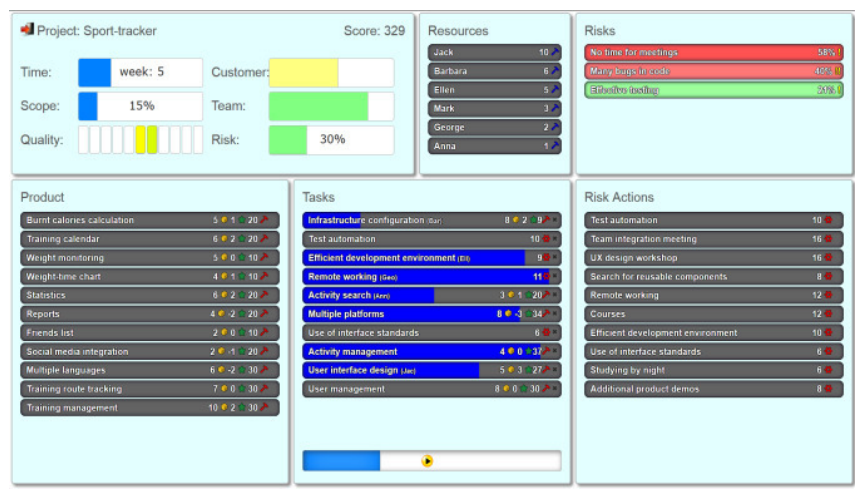

Fig. 1 The GraPM game user interface mid-game

The user experience goals for the GraPM game assume that the player understands the game mechanics after the $2^{\text {nd }}$ gameplay and the game logic after the $5^{\text {th }}$ gameplay. The player's engagement should be maintained high across multiple gameplays to ensure game replayability.

The game is also expected to generate the following emotions desired for educational effectiveness: (a) interest eagerness to learn; (b) slight confusion - feeling there is something to learn; (c) joy - satisfaction from learning and playing better; (d) feeling of control - the confidence of fully controlling the in-game project and winning the game. On the other hand, the following emotions are undesired for the educational effectiveness and should be avoided in the gameplay: (a) fear - being afraid of controlling the game and learning; (b) strong confusion (frustration) - feeling lost due to not knowing how to play the game; (c) anger - irritation due to not understanding the game and inability to win; (d) boredom - loss of motivation to play, learn and win; (e) disregard - considering the game poor, unchallenging and non-educating.

\section{USER EXPERIENCE STUDY DESIGN AND EXECUTION}

\section{A. UX Study Design}

In the study of the user experience of GraPM, we have used typical scenario-based procedure for usability testing. The procedure was performed and analyzed using the Affective-Behavioral-Cognitive (ABC) approach, that combines participant perception (cognitive component retrieved by self-report) with observational scores (behavioral component) and emotion analysis techniques (affective component). The study was a part of an experiment that aimed at evaluation of applicability of automatic emotion recognition in the context of usability testing [20]. It was performed at the Emotion Monitor stand, which is a multi-modal setting designed for human-computer interaction observation [21].

An initial questionnaire was filled before playing the game. It covered: sex, age, year and field of study, knowledge of the GraPM game or other project management games, participation in project management courses and evaluation of initial project management knowledge (IPMK).

The gameplay (which was performed 5 times) was intertwined with questionnaires that included: subjective competence progress evaluation and self-report on emotions. The questionnaires covered cognitive component of the ABC approach. In order to perform behavioral analysis, the Morae Recoder software was used, that allowed to capture screen and user mouse/keyboard activities.

Affective component used in this study was based on three input channels: video capturing for facial expressions analysis (with Logitech Internet camera and compatible software solution), self-report based on PAD (PleasureArousal-Dominance) emotion representation model [22] and physiological signals recording as a reference (skin conductance, blood-volume pulse and respiration rate recorded with ProComp Infiniti hardware coder together with Biograph software).

\section{B. UX Evaluation Criteria}

We have applied three general UX evaluation criteria: understanding, engagement and enjoyment, as well as purposely added educational effectiveness. All evaluation criteria were further operationalized into metrics.

Understanding covers the cognitive aspect of user experience and describes the comprehension of the game by the player on the level of its mechanics and its internal logic. The understanding of game mechanics means that the player is able to manipulate the game and affect the gameplay. The understanding of game logic means that the player learned the rules of the game and is able to win. According to the user experience goals of GraPM, the understanding of the 
game mechanics should be evaluated after the $2^{\text {nd }}$ gameplay, while the understanding of the game logic should be evaluated after the $5^{\text {th }}$ gameplay.

Engagement is related to the behavioral aspect of the player-game interaction and characterizes the ability of the game to attract the player, maintain his interest and motivate him to play, learn and win. It should be evaluated over several gameplays.

Enjoyment covers the emotional aspect of the user experience and describes to what extend the game is able to stimulate desired emotions and avoid undesired emotions, as defined by the game designer. It should also be evaluated over multiple gameplays.

The educational effectiveness corresponds to the main business goal of the GraPM game. The other factors of user experience form, in our opinion, an indispensable foundation for the educational effects to emerge. The educational effects should be evaluated after the $5^{\text {th }}$ gameplay.

\section{Operationalization of UX Evaluation Criteria}

The understanding factor was operationalized with two metrics: understanding of game mechanics (UM) and understanding of game logic (UL). UM measured the number of mechanics understood by the player out of 15 mechanics. UL measured the number of game logic rules understood out of 10 rules in total. The values of these metrics were measured by the game designer based on the recorded gameplay.

The engagement factor was operationalized with the following metrics: (EN1) number of pauses to adjust the project; (EN2) percentage of risks to which player reacted in any way; (EN3) number of threats eliminated (reduced to zero); (EN4) number of opportunities enhanced or exploited (materialized); (EN5) number of tooltips read; (EN6) number of mouse clicks (left button only); (EN7) distance of mouse movement (in thousands of pixels); (EN8) gameplay duration (in minutes); (EN9) summary evaluation of engagement based on EN1-EN8 in a 5-point Likert-type scale (very low VL, low $\mathrm{L}$, medium $\mathrm{M}$, high $\mathrm{H}$, very high $\mathrm{VH})$. The EN1-EN8 metrics were measured in an open scale of intensity. Metrics EN1-EN5 were measured by the game designer based on the recorded gameplay. Metrics EN6-EN8 were measured automatically by Morae Manager tool. EN9 was evaluated by the game designer.

Enjoyment factor was operationalized with the following metrics: (EJ1) average level of valence per user, representing positive vs. negative dimension; (EJ2) average level of arousal per user, representing calm vs. energetic dimension; (EJ3) compatibility of the EJ1 and EJ2 with desired or undesired emotional states in a closed scale (D - desired emotional state; UD - undesired emotional state, $\mathrm{O}$ - other emotional state than specified); (EJ4) dominance after $5^{\text {th }}$ gameplay, which is the proxy for the feeling of control.

Educational effectiveness was operationalized with the following metrics: (ED1) self-reported degree of improvement in the project management competencies in 5point Likert-type scale, from 1 - "very low" to 5 - "very high"; (ED2) number of additional unique project management aspects listed by the player after 5 gameplays as compared to the initial questionnaire.

\section{UX Study Execution}

The study was carried out in April and May 2016. The entire experiment involved 10 participants aged 23 to 43 (8 of them belonged to the game target group), 5 male and 5 female. 5 participants were selected for the user experience study based on the following criteria: (1) belonging to the target group; (2) no prior playing GraPM; (3) diverse fields of study; (4) inclusion of both male and female; (5) diverse levels of initial project management knowledge. In the entire experiment we recorded 50 gameplays, 5 for each participant. The time of a single participant recording (5 gameplays and the questionnaires) varied from 34 up to over 90 minutes. The players in the study are coded P1 to P5.

\section{V.USER EXPERIENCE EVALUATION RESULTS}

\section{A. Understanding}

The understanding of the game mechanics and logic by the sample players are presented in Table I. It can be observed that, apart from player P1, the players understood at least $80 \%$ of the game mechanics only after the $2^{\text {nd }}$ gameplay. The results also show that 2 out of 5 players (P1 and P4) understood at most $50 \%$ of the game logic, while the other 3 at least $80 \%$. The players P1 and P4 were not able to win the game as they had considerable usability problems only at the game mechanics level. Only 2 players (P3 and P5) could achieve full project success and win the game.

TABLE I.

EVALUATION OF UNDERSTANDING

\begin{tabular}{|l|l|l|l|l|l|l|}
\hline Metric & \multicolumn{1}{|c|}{ P1 } & \multicolumn{1}{|c|}{ P2 } & \multicolumn{1}{|c|}{ P3 } & \multicolumn{1}{c|}{ P4 } & \multicolumn{1}{|c|}{ P5 } & \multicolumn{1}{c|}{ Avg. } \\
\hline UM & 8 & 13 & 15 & 12 & 13 & $12,2(2,6)$ \\
\hline UL & 5 & 8 & 9 & 4 & 9 & $7(2,3)$ \\
\hline
\end{tabular}

\section{B. Engagement}

The results of the engagement evaluation are presented in Table II. The values of metrics EN1 to EN5 were averaged over the $2^{\text {nd }}$ and the $5^{\text {th }}$ gameplay, while the values of metrics EN6 to EN8 were averaged over all 5 gameplays of a particular player.

Interpreting these results, we can see that the least engaged player was P4. P4 influenced the project course to a very limited extent (EN1), generally ignored the risks (EN2EN4), clicked and moved the mouse little (EN6, EN7) as well as played for the shortest time (EN8). The most engaged player was P3. P3 adjusted the project many times (EN1), managed successfully the risks (EN2-EN4), read many tooltips (EN5), intensely clicked and moved the mouse (EN6, EN7), and played for the longest time (EN8). The 
engagement of P2 was also very high, similar to P3. The P4's engagement was high, close to the average. The anomalous case is the player P1. P1 did not understand how to pause the game (EN1) and react to risks (EN3 and EN4) on the game mechanics level (compare to Table I), thus the 0 values of these metrics. However, P1's engagement was at least medium, which can be evaluated from the metrics EN2, and EN6 to EN8. These metrics show that P1 wanted to control the project and manage the risks, but did not know how to manipulate the game to do this. These interpretations are reflected in the values assigned to the metric EN9.

TABLE II.

EVALUATION OF ENGAGEMENT

\begin{tabular}{|l|l|l|l|l|l|l|}
\hline Metric & \multicolumn{1}{|c|}{ P1 } & \multicolumn{1}{c|}{ P2 } & \multicolumn{1}{c|}{ P3 } & \multicolumn{1}{c|}{ P4 } & \multicolumn{1}{c|}{ P5 } & \multicolumn{1}{c|}{ Avg. } \\
\hline EN1 & 0 & 12,5 & 25,5 & 1 & 14 & $10,6(10,5)$ \\
\hline EN2 & $80 \%$ & $100 \%$ & $100 \%$ & $42 \%$ & $72 \%$ & $78,7 \%(24 \%)$ \\
\hline EN3 & 0 & 3,5 & 3,5 & 0,5 & 2 & $1,9(1,6)$ \\
\hline EN4 & 0 & 1,5 & 1,5 & 0 & 1 & $0,8(0,8)$ \\
\hline EN5 & 0 & 0 & 7 & 4 & 0 & $2,2(3,2)$ \\
\hline EN6 & $\begin{array}{l}50 \\
(14,9)\end{array}$ & $\begin{array}{l}87 \\
(6,2)\end{array}$ & $\begin{array}{l}108 \\
(31,3)\end{array}$ & $\begin{array}{l}17 \\
(5,0)\end{array}$ & $\begin{array}{l}73 \\
(11,8)\end{array}$ & $\begin{array}{l}67,2 \\
(34,9)\end{array}$ \\
\hline EN7 & $\begin{array}{l}59,0 \\
(16,9)\end{array}$ & $\begin{array}{l}97,5 \\
(21,4)\end{array}$ & $\begin{array}{l}90,8 \\
(18,0)\end{array}$ & $\begin{array}{l}30,7 \\
(14,1)\end{array}$ & $\begin{array}{l}75,8 \\
(8,6)\end{array}$ & $\begin{array}{l}70,8 \\
(26,9)\end{array}$ \\
\hline EN8 & $\begin{array}{l}5: 44 \\
(1: 26)\end{array}$ & $\begin{array}{l}6: 32 \\
(1: 26)\end{array}$ & $\begin{array}{l}8: 32 \\
(2: 40)\end{array}$ & $\begin{array}{l}4: 29 \\
(0: 38)\end{array}$ & $\begin{array}{l}6: 24 \\
(0: 35)\end{array}$ & $\begin{array}{l}6: 20 \\
(1: 28)\end{array}$ \\
\hline EN9 & M & VH & VH & L & H & H \\
\hline
\end{tabular}

\section{Enjoyment}

The evaluation of enjoyment in 5 gameplays for each player is presented in Table III and Fig. 2.

TABLE III.

EVALUATION OF ENJOYMENT

\begin{tabular}{|l|l|l|l|l|l|}
\hline \multicolumn{1}{|c|}{ Metric } & \multicolumn{1}{c|}{ P1 } & \multicolumn{1}{c|}{ P2 } & \multicolumn{1}{c|}{ P3 } & \multicolumn{1}{c|}{ P4 } & \multicolumn{1}{c|}{ P5 } \\
\hline EJ1 & $3,6(0,9)$ & $6,2(0,4)$ & $5(0)$ & $5,4(1,3)$ & $6,4(0,5)$ \\
\hline EJ2 & $3,8(0,4)$ & $5,6(1,1)$ & $1(0)$ & $5,2(0,8)$ & $2,4(1,7)$ \\
\hline EJ3 & D & D & O & D & O \\
\hline EJ4 & 5 & 5 & 5 & 3 & 7 \\
\hline
\end{tabular}

Desired emotional states region contains majority of the $1^{\text {st }}$ quarter together with the states of neutral arousal from the negative region. Out of the four specified desired emotional states (section III), two are not expressible in the valence-arousal model: interest as a cognitive state and a feeling of control, which is expressible with the third dimension of PAD model - dominance (EJ4). Undesired emotional states were clustered as two regions: negative states of very high arousal and negative states of very low arousal. Please note, that fear, anger and frustration are very close to each other in this model.

The players P1, P2 and P4 fall into the desired emotional states region. The players P3 and P5 do not fall into the desired nor un-desired region; it seems, that for them the game was a relaxing experience.

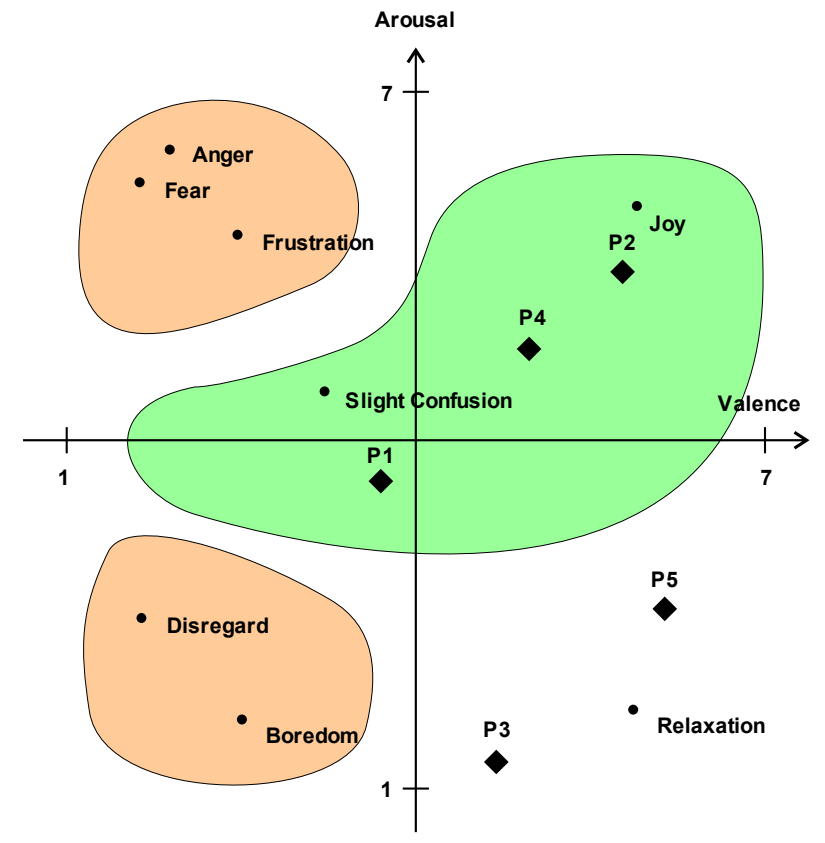

Fig. 2 Players' emotional states compared to the desired and undesired emotional state regions in the valence-arousal model

\section{Educational Effectiveness}

The evaluation of the educational effectiveness after the $5^{\text {th }}$ gameplay is presented in Table IV. The values of metrics ED1 and ED2 are confronted with the initial player's knowledge of project management before playing the GraPM game (IPMK). It can be observed that the players P2 and P5 reported the highest educational effectiveness, however in case of P5 the high self-reported estimate (ED1) was not confirmed with the knowledge verification (ED2). P3 reported low estimate of education (ED1), but still exhibited considerable increase in knowledge (ED2). The lowest educational effectiveness was observed for P1 and P4.

\section{TABLE IV.}

EVALUATION OF EDUCATIONAL EFFECTIVENESS

\begin{tabular}{|l|l|l|l|l|l|l|}
\hline \multicolumn{1}{|c|}{ Metric } & \multicolumn{1}{|c|}{ P1 } & \multicolumn{1}{c|}{ P2 } & \multicolumn{1}{c|}{ P3 } & \multicolumn{1}{c|}{ P4 } & \multicolumn{1}{c|}{ P5 } & \multicolumn{1}{c|}{ Avg. } \\
\hline ED1 & 2 & 4 & 2 & 1 & 4 & $2,6(1,3)$ \\
\hline ED2 & 1 & 3 & 3 & 2 & 1 & $2(1,0)$ \\
\hline IPMK & 3 & 4 & 5 & 2 & 1 & $3(1,6)$ \\
\hline
\end{tabular}

\section{E. Summary and Discussion of Results}

This study leads to the following observations:

- the understanding of the game mechanics forms the basis for understanding of the game logic;

- the engagement seems unrelated to understanding of the game mechanics, which can be seen by cases of player P1 (at least medium engagement with low understanding of the mechanics) and player P4 (low engagement with high understanding of the mechanics);

- the engagement is related to understanding of the game logic, however the nature of this relation is still to be investigated; 
- the educational effectiveness is enhanced by the player's understanding of the game mechanics and logic, as well as his engagement (P2, P3, and P5);

- the educational effectiveness seems unrelated to the initial knowledge of the player: players P2 and P3 reported the highest initial knowledge but still exhibited high educational effects; player P5 reported the lowest initial knowledge and high educational effectiveness; players P1 and P4 reported medium initial knowledge and medium educational effects;

- the educational effectiveness was not disturbed with undesired emotions for any player, however 2 out of 3 players (P3 and P5) exhibited rather ambivalent emotions regarding the game goals (relaxation);

- the educational effectiveness is related to the player's feeling of control: the player P4 with the lowest educational effects also had the lowest feeling of control, while the player P5 with high educational effects exhibited the highest feeling of control.

We are aware of the fact that the validity of this study has some limitations. We identified and addressed the following threats to its validity: (1) sample size - we engaged 5 users as the usability tests show that 5 users reveal $75-90 \%$ of the usability issues; (2) sample as a group of convenience - we introduced the initial questionnaire and selected the sample for UX evaluation to ensure its diversity; (3) confounding variables - we performed the study in a strictly controlled environment, where we limited the possible influences of external factors; (4) subjective measurements - we operationalized most of the variables to objective metrics, the number of subjective self-reports is minimal; (5) small number of gameplays - due to resource limitations in manual measurements we focused on $2^{\text {nd }}$ and $5^{\text {th }}$ gameplay, which were specified in the UX design goals.

\section{CONCLUSION}

The study provides preliminary evidence that usability and user experience affect the educational effectiveness of a computer game. The results revealed the flaws in the GraPM game UX design at the cognitive and behavioral levels. Together with the analysis of the emotional impact it may be used to improve the game and increase its effectiveness.

Based on the data collected we plan to evaluate the GraPM learning curve based on the understanding of logic across gameplays, analyze players' emotional state in consecutive gameplays as well as identify affects related to particular game events.

\section{ACKNOWLEDGMENT}

J. Miler thanks the engineering diploma project team that implemented the GraPM game: Rafał Piechowski, Damian Płatek, Anna Wasik, and Sylwia Grabowska. The authors thank Dominika Makowiecka, who helped in the experiment setting and execution.

\section{REFERENCES}

[1] L. W. Anderson, D. R. Krathwohl, P. W. Airasian, K. A. Cruikshank, R. E. Mayer, P. R. Pintrich, J. Raths, and M. C. Wittrock, A Taxonomy for Learning, Teaching, and Assessing: A Revision of Bloom's Taxonomy of Educational Objectives, Abridged Edition, Pearson, 2000

[2] C. Abt, Serious Games, University Press of America, 2002

[3] D. Novick, J. Vicario, B. Santaella, I. Gris, "Empirical analysis of playability vs. Usability in a computer game," LNCS vol. 8518, PART 2, Springer Verlag, 2014

[4] ISO 9241-210:2010 Ergonomics of human-system interaction -- Part 210: Human-centred design for interactive systems, ISO, 2010.

[5] ODiTK Symulator Biznesu Sp. z o.o., http://www.symulator.oditk.pl/ [retrieved May 2016]

[6] R. Charney, That Project Management Game, http://thatpmgame.com/ [retrieved May 2016]

[7] A. Gkritsi, "Scrum Game: An Agile Software Management Game," M.S. thesis, School of Electronics and Computer Science, Univ. of Southampton, Southampton, UK, 2011.

[8] K. Isbister, and N. Schaffer, Game Usability: Advancing the Player Experience, CRC Press, 2008

[9] P. Mirza-babaei, "Biometrics to improve methodologies on understanding player's gameplay experience," in Proc. 25th BCS Conference on Human-Computer Interaction, Swinton, UK, 2011, pp. 546-549.

[10] E. Parodi, M. A. Bedek, P. Seitlinger, M. Vannucci, C. Jennett, M. Ruskov, and J. M. Celdran, "Analysing players' performance in serious games", International Journal of Technology Enhanced Learning (IJTEL), vol. 6, no. 3, 2014, pp. 237-248.

[11] A. Raabe, E. Santos, L. Paludo, and F. Benitti, "Serious Games Applied to Project Management Teaching,", in Handbook of Research on Serious Games as Educational, Business and Research Tools, IGI Global, Hershey, PA, 2012, pp. 668-692.

[12] A. P. Vermeeren, E. L. C. Law, V. Roto, M. Obrist, J. Hoonhout, and K. Väänänen-Vainio-Mattila, "User experience evaluation methods: current state and development needs," in Proc. 6th Nordic Conference on Human-Computer Interaction: Extending Boundaries, ACM, 2010, pp. 521-530.

[13] W. Albert and T. Tullis, Measuring the user experience: collecting, analyzing, and presenting usability metrics, Newnes, 2013

[14] H. I. Ahn and R. Picard, "Measuring Affective-Cognitive Experience and Predicting Market Success", IEEE Transactions on Affective Computing, vol. 5, no. 2, 2014

[15] P. Lew, L. Olsina, P. Becker, and L. Zhang, "An integrated strategy to systematically understand and manage quality in use for web applications," Requirements Engineering, vol. 17, no. 4, 2012, pp. 299-330.

[16] A. Kołakowska, A. Landowska, M. Szwoch, W. Szwoch, M. Wróbel, "Emotion recognition and its applications", Human-Computer Systems Interaction: Backgrounds and Applications 3, Springer, 2014, pp. 51-62.

[17] J. Miler and H. Wesołowska, "Improvement of Task Management with Process Models in Small and Medium Software Companies," in Proc. $19^{\text {th }}$ EuroSPI Conference, CCIS Systems, Software and Services Process Improvement, vol. 301, Springer, 2012, pp. 145-156.

[18] J. Miler, "A Method of Software Project Risk Identification and Analysis," Ph. D. thesis, Faculty of Electronics, Telecommunications and Informatics, Gdansk Univ. of Techn., Gdansk, Poland, 2005.

[19] R. Piechowski, D. Płatek, A. Wasik, S. Grabowska, "Educational game on project management", B.Sc. thesis, Faculty of Electronics, Telecommunications and Informatics, Gdansk Univ. of Techn., 2014.

[20] A. Landowska, J. Miler, "Limitations of Emotion Recognition in Software User Experience Evaluation Context," in Proc. of FedCSIS, 2016, accepted for publication

[21] A. Landowska, "Emotion monitor-concept, construction and lessons learned," in Proc. of Federated Conference on Computer Science and Information Systems (FedCSIS), 2015, pp.75-80.

[22] A. Kołakowska, A. Landowska, M. Szwoch, W. Szwoch, M. R. Wróbel, "Modeling emotions for affect-aware applications," in Wrycza S. Information Systems Development and Applications, 2015, pp. 55-64. 